Chemotactic behaviors in eukaryotic cells such as cellular slime molds (Dictyostelium descoideum) and other animal cells are widely known phenomena Though a straight motion toward the chemical source can be observed in chemotaxis, cells do not necessarily move in a straight way but often move in fluctuated zigzag ways. For understanding such variety of behaviors, we build simple model for chemotactic eukaryotic cells, which describes changes in cell shape on the two-dimensional plane by considering a cell membrane, actin filaments embedded in the membrane, and an intracellular control factor. We also introduce three models of environment around cells: (a) simple chemical gradient in which the chemical guidance (chemotactic signal) spreads everywhere in two-dimensional field with a uniform gradient; (b) flipping chemical gradient with which the uniform gradient is reversed at a moment everywhere in the space and (c) a maze around a chemical source, in which maze-like walls separate the source and cells to block cell's locomotion. Some parts in those walls permeate the chemical guidance to confuse cells. The simulated results show that in the case (a) cell's behavior is most efficient when the cell takes a crescent shape like "keratocyte" and moves in a straight way. In contrast, in cases of (b) and (c) the behavior is efficient when the cell takes a typical amoeboid-like shape and moves in zigzag ways. These results suggest that the fluctuated zigzag locomotion of amoeboid cell has an advantage in some "complex" environment.

\section{TA5-01 タンパク質平衡摇らぎの独立成分分析}

2P-064 Independent Component Analysis of Equilibrium Fluctuations in Proteins

Yusuke Naritomi (1), Sotaro Fuchigami (1), Mitsunori Ikeguchi (1), Akinori Kidera (2) ((l) Supramolecular Biology, International Graduate School of Arts and Sciences, Yokohama Cit University: (2) Supramolecular Biology, International Graduate School of Arts and Sciences, Yokohama City University; Research Program for Computational Science, RIKEN)

Protein fluctuations in equilibrium are an important factor for its conformational change and function. It has been found that a small number of low-frequency modes in a protein, determined by normal model analysis or principal componen analysis, account for its large fluctuations and contribute significantly to its conformational change. However, dynamical aspects of protein fluctuations and their roles in protein function remain unclear. In the present study, we attempt to characterize dynamical properties of protein fluctuations in equilibrium by using molecular dynamics simulation and independent component analysis (ICA), and to reveal their roles in protein conformational change based on time-dependen linear response theory (TDLRT). As a target protein, we chose lysine/argineine/ornithine-binding protein (LAO), which undergoes a large conformational change upon ligand binding. A $300 \mathrm{~ns}$ molecular dynamics simulation of apo-LAO in explicit water was performed using MARBLE and the CHARMM22/CMAP force field parameters. ICA was applied to an equilibrated part of the trajectory, obtaining independent component (IC) modes of LAO which represent protein motions on nanosecond timescale. Differences between IC modes and principal component modes are discussed. Finally, a time course of conformational change of LAO described by TDLRT is examined by using IC modes in order to elucidate roles of protein fluctuations on nanosecond timescale in its conformational change.

\section{TA5-02 ベイズ法によるタンパク質の内部構造の推定}

2P-065 Uncovering the hidden structure of the protein using Bayesian inference

Makito Miyazaki (1), Takahiro Harada (2) ((1) Department of Physics, Graduate School of Science, Kyoto University: (2) Department of Physics, Graduate School of Science, The University of Tokyo)

In single-molecule experiments of molecular motors, attaching a large probe particle is a widely adopted strategy to visualize the motion of the molecule. In this case, the observable variables are restricted within a small fraction of the whole degrees of freedom. Therefore, we need to estimate an internal structure of a protein, i.e. the system parameters, only referring to the motion of accessible degrees of freedom. To overcome this problem, we employed Bayesian framework and developed a new method applicable for various complex systems including nonlinear interactions. At first, by assuming the basic structure of the system, the path probability of the system can be calculated. Then, we integrated out the invisible degrees of freedom and obtained a marginal likelihood. In the limit of an infinite observation time, we obtained the general evidence: maximization of the marginal likelihood leads to the correct estimates of the system parameters. We also examined a practical utility of our method in the case of finite-time observation. From careful numerical studies using a simple model, we confirmed that the performance of our method is as well as or better than the conventional method. Furthermore, we found that there is a critical line in the original parameter space below which the precision of the estimate is abruptly lost. We will report the effectiveness of our method including the property of "loss-ofprecision" transition and applications for a single molecule experiment.

2TA5-03 低温電子顕微鏡法における生体超分子複合体に対する電子線照射 2P-070 損傷の定量的評価
Electron dose effect on 3D image reconstruction of a biological macromolecular structure by electron cryomicroscopy

Masamichi Ashihara (1), Takayuki Kato (1), Takashi Fujii (1), Hideyuki Matsunami (2), Tomoko Miyata (1), Kenji Iwasaki (3), Keiichi Namba (1) ((1) Graduate School of Frontier Biosciences, Osaka University: (2) Okinawa Institute of Science and Technology: (3) Institute for Protein Research, Osaka University)

The principal limitation in achieving high resolution by electron cryomicroscopy of frozen-hydrated biological specimen is the extremely low contrast and signalto-noise-ratio (SNR) due to a low electron dose to avoid radiation damage. Reduction of the radiation sensitivity would be the simplest way to improve the resolution limit. It has been demonstrated that cooling specimens to $4 \mathrm{~K}$ with liquid helium reduces the damage and allows statistically better images to be recorded. In fact an atomic model of the bacterial flagellar filament has been built from images recorded at $4 \mathrm{~K}$. However, 3D image reconstructions by single particle image analysis at resolution of $5 \AA$ or better from images recorded at $100 \mathrm{~K}$ have recently been reported. We therefore decided to reexamine the advantages of liquid-helium cooling over higher specimen temperatures to determine the optimal specimen temperature for high-resolution image recording. We compared 3D density maps of the Hepatitis B virus core particle reconstructed from images recorded at different temperatures and accumulated dose levels to examine the temperature effect on radiation damage. We found no significant difference in resolution between $4 \mathrm{~K}$ and $50 \mathrm{~K}$ at $20 \mathrm{e}^{-} / \AA^{2}$. However, the density maps of the virus from images recorded at $50 \mathrm{~K}$ were significantly better in both resolution and quality than those at $4 \mathrm{~K}$ when the dose was accumulated further, indicating that cooling specimens down to $4 \mathrm{~K}$ is disadvantageous for electron cryotomography at relatively high dose levels.

\section{TA5-04 誘電緩和分光による酸性から中性 pH 領域における} Pseudomonas aeruginosa シトクロム $\mathrm{c}$ の水和解析

Hydration study of Pseudomonas aeruginosa cytochrome $c 551$ in the range of acidic to neutral $\mathrm{pH}$ by dielectric relaxation spectroscopy

Tetsuichi Wazawa (1), Takashi Miyazaki (2), Takafumi Sonoyama (3), Yoshihiro Sambongi (3), Makoto Suzuki (2) ((1) CREST, JST: (2) Tohoku University Graduate School of Engineering: (3) Graduate School of Biosphere Science, Hiroshima University)

Hydration is an essential factor that governs the conformation, dynamics and function of proteins. Dielectric relaxation spectroscopy (DRS) is one of the most powerful techniques for analyzing protein hydration. To investigate the correlation of the hydration of Pseudomonas aeruginosa cytochrome c551 (PA c551) with its conformation, we analyzed the dielectric relaxation (DR) spectrum of aqueous PA c551 solution in the acidic to neutral $\mathrm{pH}$.

A $\mathrm{pH}$ titration of ferric PA c551 monitoring the absorbance in visible wavelengths showed that the acid-denaturation occurred through a one-step reaction with pKa and Hill coefficient of 1.84 and 2.05 , respectively, at $20^{\circ} \mathrm{C}$. Furthermore, the DRS of aqueous PA c551 solution was performed on a network analyzer over the $\mathrm{pH}$ range of $1.0-6.2$ at $20^{\circ} \mathrm{C}$. A least squares analysis of the DR spectra of PA c551 solution with Debye series was conducted to derive the microwave dielectric property of the hydration shell.

The DRS study demonstrated that the hydration shell of PA c551 exhibited a dispersion with a DR frequency of ca. $5 \mathrm{GHz}$, independent on the conformation, for $\mathrm{pH}$ 1.0-2.7. However, the DR amplitude of the hydration shell and the number of hydration water decreased by $30 \%$ and $40 \%$, respectively, upon refolding. These changes were well described by a two-state transition and perfectly consistent with the absorbance titration data. Furthermore, interestingly, the hydration water of the native PA c551 showed a slight increase of the mobility as $\mathrm{pH}$ increased from 2.7 to 6.2 , although its conformation unchanged. Thus, our DRS analysis has been proven to successfully acquire the information of the protein hydration, and furthermore, must provide important insights into protein hydration.

2TA5-05 微細加工基板上でのマイコプラズマ・モービレ運動についての改 良されたシミュレーション

An improved simulation of Mycoplasma mobile movements on micropatterned surfaces

Takahiro Nitta (1), Shogo Asano (1), Yuichi Hiratsuka (2), Makoto Miyata (3), Motohisa Hirano (1) ((1) Gifu University: (2) JAIST: (3) Osaka City University)

M. mobile, a fish pathogen, glides over solid surfaces at the speed of severa micrometers per second. $M$. mobile cells can be seen as microscale workhorses. Indeed, microrotary motors powered by $M$. mobile have been demonstrated However, the fraction of rotating micromotors was small. A possible reason for this was unsuccessful guiding of $M$. mobile movements. The guidance of $M$. mobile movements was achieved by utilizing the tendency of $M$. mobile cells to move along the edges of micropatterned surfaces. The micropatterns have to be carefully designed for $M$. mobile movements to be guided in the intended manner. However, it is difficult to design proper micropatterns by experimental trial and error. A computer simulation would be useful in designing micropatterns. We aim at developing such a simulation. In the previous study, we reported a computer simulation on $M$. mobile movements based on a random walk model having a 